\title{
Progress of breeding strategies in winter rye: from mass selection to genomic selection
}

Ponomareva M.L. ${ }^{2 *}$, Ponomarev S.N. ${ }^{1}$

${ }^{1}$ Tatar Research Institute of Agriculture, FRC Kazan Scientific Center of RAS, Kazan, Russia

${ }^{2}$ Laboratory of Plant Infectious Diseases, FRC Kazan Scientific Center of RAS, Kazan, Russia

*email: smponomarev@yandex.ru

Winter rye is great importance in the global economy and food traditions in 7 European countries, where this crop covers over more than 100 thousand hectares (Germany, Poland, Russia, Belarus, Denmark, Spain, Ukraine). According to FAOSTAT in 2019 the world rye grain production was 13 million tons, of which $81 \%$ were grown on the European continent with an average yield of $30.4 \mathrm{dt} / \mathrm{ha}$. The specificity of breeding programs in rye-growing countries including Russian Federation is due to the peculiarities of climatic conditions, soil fertility, and national traditions of consumers. The main breeding directions for the coming years remain the adaptability to unfavorable abiotic and biotic factors, increase of the yield, the targeted use of the final product and its high quality. Work in this direction has led leading winter rye producers in Europe to replace traditional population varieties with $\mathrm{F}_{1}$ hybrids on $60 \%$ of the area. In rye breeding, three main methods are used to form a new genetic pool: selection in existing populations, recombination and induction of mutations. In recent years, rye genetics has evolved rapidly. Modern genomics-based techniques, like genome-wide association and/or linkage mapping should be applied. A large number of tasks of functional and structural genetics and rye genomics are solved with molecular markers, some of which have found their application in practical breeding. For rye, genomic selection, as opposed to marker-based, is a more promising methodology for rapid improvement of quantitative plant traits. The integration of modern technologies with conventional methods in rye breeding programs has significantly accelerated the time from first hybrid to cultivar release, while maintaining the increase of average yield and improving other traits such as biotic and abiotic stress resistances as well as quality. 